\title{
Adaptation through minimization of the phase lag in coupled nonidentical systems
}

\author{
Rhonda Dzakpasu and Michał Żochowski ${ }^{\text {a) }}$ \\ Department of Physics and Biophysics Research Division, University of Michigan, \\ Ann Arbor, Michigan 48109
}

(Received 4 December 2003; accepted 21 May 2004; published online 24 August 2004)

\begin{abstract}
We show that the internal control of adaptation can be obtained from the properties of the phase lag that results from phase synchronization of two nonidentical chaotic oscillators. The direction and magnitude of the phase lag depend upon the relative internal properties of the coupled units, and they can be used as indicators during the adjustment of dynamics, i.e., adaptation of the target unit to match that of the control. The properties of the phase lag are obtained using a method based on the estimation of properties of the distributions of relative event times of both (target and control) units. The phase lag dependent mechanism to control the adaptation process was applied to a system of nonidentical Rössler oscillators and a system of nonidentical Lorenz oscillators. We also elucidate its importance as a control mechanism of the changes of neuronal activity showing its application to neural adaptation. (C) 2004 American Institute of Physics. [DOI: 10.1063/1.1772171]
\end{abstract}

We present here a novel parameter adaptive control technique, allowing for synchronization of a system of two initially nonidentical units. The mechanism of adaptation is based on a measurement of conditional entropies that utilizes the properties of temporal distribution of interevent intervals of the coupled units. Those properties are driven by the phase lag observed during phase synchronization of the two units, which is measured by monitoring a relative delay in the generation of an event by one unit with respect to the other unit. The properties of this phase lag depend on the relative frequencies of the two units and thus can be used as an indicator during the adaptation process. The major advantages of the presented adaptation mechanism are that it depends only on the relative timings of the events generated by the units that are often possible to obtain experimentally and it does not contain any information about the mathematical form of the system. We show the workings of the proposed mechanism for a system of two coupled, nonidentical Rössler and Lorenz oscillators. We show that this parameter adaptive technique works well over a wide range of parameters of the two units as well as in the presence of noise. In the last part of the paper we also argue that a similar mechanism, based on relative spike timings, could be used to control the adaptation in the nervous system and as an example we demonstrate its application to the system of coupled Hindmarsh-Rose models of the thalamocortical neurons.

\section{INTRODUCTION}

Synchronization of coupled identical systems has been studied extensively during recent years and it is well known

\footnotetext{
${ }^{a)}$ Electronic mail: michalz@umich.edu
}

that with the application of the appropriate coupling, the identical systems will achieve complete synchronization, ${ }^{1-7}$ which is often observed in nature. ${ }^{8-13}$

However in many cases even though the dynamics of the coupled units are driven by the same underlying processes (i.e., it is described by the same structural equations), the macroscopic properties of their trajectories will be dramatically different and will depend upon the sets of control parameters. Such systems may synchronize only after the coupled units undergo an adaptation process to make their trajectories compatible. This is well exemplified in neural systems where the generation of action potential by neurons is driven by appropriate temporal changes in ionic currents, but it is known that the receptor and ion channel repertoire of different neuron types are not the same and thus the specific properties of action potentials (i.e., spike threshold, existence of self slow excitation and inhibition currents, differences in refractory time, etc.) will vary. Moreover, it has been determined experimentally that the neurons can change their firing pattern dramatically by altering their ionic currents depending on the cognitive state of the animal ${ }^{14}$ and thus indicating radical changes in its internal properties.

Parameter adaptive control techniques have been investigated by others but these techniques usually require construction of the Liapunov functions based on the structural information about synchronizing units. ${ }^{15-17}$ This information may often not be readily available and is definitely not available to drive the adaptation in dynamical systems found in nature.

We have shown earlier that the parameter adaptive control of two initially nonidentical units can be achieved by monitoring the changing differences of the spatial properties of the trajectories of both units. ${ }^{18}$ The mechanism of parameter adaptive control in that case was constructed from the distribution of the spatial coordinates of the Poincare section crossing was changing monotonically with the control parameter of the system. However, a question that becomes 
pertinent is whether and how the temporal information about the interaction of coupled nonidentical systems can be used to change their dynamical properties (i.e., induce an adaptation process) so that they can converge to similar dynamical trajectories in a self-controlled manner, i.e., self-organize to form a spatio-temporal response pattern.

Here we show that the parameter adaptive control can be achieved based on temporal interdependencies of the interevent timings of the two, coupled units. Namely, we use the fact that the properties of the phase lag achieved during phase synchronization depend on the relative frequencies of the coupled units. We monitor the properties of the phase lag using a measure based on calculation of conditional entropies $^{19}$ and use the differences reported by that measure as a driving mechanism during the adaptation process (i.e., adjustment of control parameter of the target unit). The adaptation is thus based solely on the relative temporal properties of the coupled units and does not require any information about underlying equations of the system nor specific properties of the units trajectories. This fact makes it applicable to many experimental systems as it is often possible to detect and monitor event timings but not the unit trajectories themselves.

We show applicability of the method for two different systems: (1) coupled nonidentical Rössler oscillators ${ }^{20}$ and (2) coupled nonidentical Lorenz oscillators. ${ }^{21}$ In Sec. III we link this adaptation mechanism to neural activity by showing that the same phenomenon can be observed in coupled, nonidentical Hindmarsh-Rose models of thalamocortical neurons. ${ }^{22-24}$ Finally, we show that this control mechanism can also be applied to control the dynamics of two neurons and may lead to the formation of the complete synchronized state observed in the brain. ${ }^{8,9,13}$

\section{MONITORING PROPERTIES OF THE PHASE LAG DURING THE PHASE SYNCHRONIZATION}

It has been established that a periodically driven nonlinear oscillator or a system of coupled, nonidentical oscillators can achieve phase synchronization. ${ }^{7,25-29}$ Here, to establish the adaptation mechanism, we use the fact that the phase lag achieved during phase synchronization will depend critically on the relative properties (i.e., relative frequencies) of the coupled units. $^{25,26}$

To measure the phase lag, we have converted the phase interdependencies of the coupled units into relative interevent intervals that correspond to specific phase differences between the two. Such formulation may be particularly useful when the timing of the event defining the phase can be measured experimentally-i.e., the spike of a neuron, or in the case of the Lorenz and Rössler oscillators the timings of trajectory of the unit crossing a defined Poincaré section.

We applied the measure based on dynamic evaluation of conditional entropies ${ }^{19}$ to monitor changing distributions of the relative interevent intervals of the two units. The distributions are created for every unit and simultaneously keep track of relative lengths of the interevent timings of unit 2 with respect to the events of unit 1 and, unit 1 with respect to the timings of unit 2. Specifically, the interevent intervals

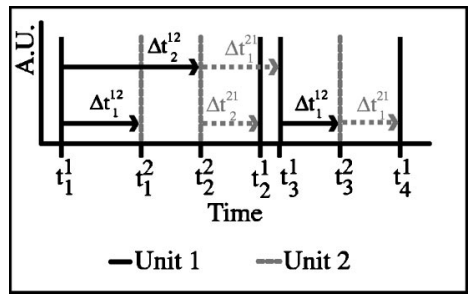

FIG. 1. Relative interevent lengths used to calculate the conditional entropy. The interevent intervals of one unit are calculated with respect to the timing of the last event of the other one. This assures asymmetry of the measure with respect to the sign of the relative phase shift between the two units.

$\Delta t_{i}^{12}$ of unit 2 with respect to unit 1 are calculated as a time difference from the last event of unit 1 , and conversely the interevent intervals $\Delta t_{i}^{21}$ of unit 1 are calculated as a time difference with respect to the timing of the last event of unit 2 (Fig. 1). Thus, if $t_{1}^{1}, t_{2}^{1}$ are the event timings of unit one, $t_{1}^{2}, t_{2}^{2}$ are the event timings of unit 2 , and if $t_{1}^{1}>t_{1}^{2}>t_{2}^{1}>t_{2}^{2}$, then one distribution will be updated only with one interevent value $\left(\Delta t^{21}=t_{2}^{1}-t_{1}^{2}\right)$, whereas the second distribution will be updated twice with $\left(\Delta t^{12}=t_{1}^{2}-t_{1}^{1}\right)$ and $\left(\Delta t^{12}=t_{2}^{2}\right.$ $\left.-t_{2}^{1}\right)$. The distributions defined in that way are complementary to each other and provide full information about timing interdependencies of both units.

The distributions are updated throughout the simulation to allow monitoring of the changes of the relative phases of the events. The distributions are updated by increasing the bin of the appropriate distribution within which the latest interevent length falls, by a fixed $\Delta P$. Specifically, if the $n$th event occurs at unit 1 at a time $t_{n}$ so that the relative interevent length $\Delta t_{n-1}^{21}$ (measured from the last event of unit 2) is of the interval $\Delta T(I-1)<\Delta t_{n-1}^{21} \leqslant I \Delta T$ and it falls within the bin $I$, then the cumulative probability of bin $I$ of the distribution of the interevent lengths of unit 1 with respect to those of unit 2 at $t_{n}$ is given by

$$
P_{I}\left(t_{n}\right)=P_{I}\left(t_{n-1}\right)+\Delta P,
$$

and the updated distribution is then renormalized.

To determine the properties of the two distributions we calculate their entropies $S=-\Sigma_{I} P_{I} \ln P_{I}$. The value of the entropy will depend on the direction of the phase lag when the phase synchronization of the two units is achieved. Namely, when one unit has a consistently constant phase delay with respect to the other unit (due to a phase synchronization), the distribution of relative interevent intervals of that unit with respect to the events of the other one will have a very narrow distribution and thus the entropy will tend to zero. Conversely, if the phase of one unit will systematically precede that of the other one, the interevent interval calculated with respect to that other unit will be widely distributed as the next pair of phase locked events happens after a time that varies chaotically. An example of this is shown in Fig. 2. The distribution of the interevent intervals of unit 1 which has a smaller value of control parameter with respect to that with a larger value of the control parameter (unit 2) is plotted in Fig. 2(A). The distribution is very narrowly peaked around the value of the delay of the events at unit 1 with respect to those at unit 2. This suggests that events at unit 1 systemati- 


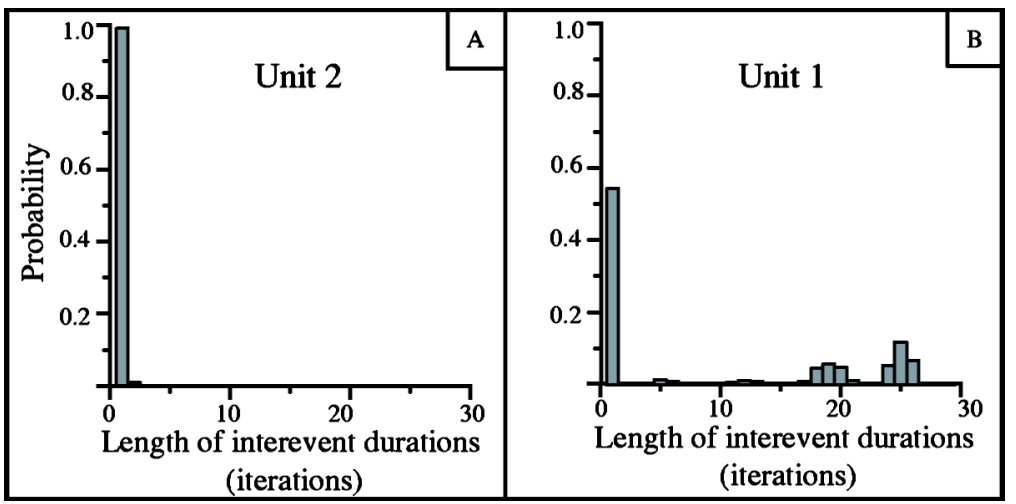

FIG. 2. Distributions of the relative interevent lengths of the two, nonidentical Rössler oscillators: (A) distribution of the interevent lengths of the unit 1 (control parameter $a_{1}=0.1$ ) with respect to latest event of the unit 2 (control parameter $a_{2}=0.3$ ) and (B) distribution of the relative interevent lengths of the unit 2 with respect to latest event of the unit 1 . The distributions are significantly different.

cally occur within the similar time interval shortly after those of unit 2. On the other hand, the distribution for the reversed situation is depicted in Fig. 2(B). In this case the distribution is significantly wider, indicating an unpredictability of the delay of the next event pair.

To show robustness of the measure, the conditional entropies based on the distributions of interevent intervals are calculated for different control parameter mismatch for both Rössler and Lorenz systems plotted in Fig. 3. In those simulations, the value of control parameter of one unit is kept constant and the simulation is repeated for different values of the control parameter of the other unit. No adaptation is present and the distributions are updated throughout 300000 iterations during which time the two nonidentical units reached a constant phase dependence. The plotted pair of values of the entropies is calculated from the distribution obtained for every simulation run. The value of conditional entropy (CE) of the unit [Fig. 3(A): Rössler oscillator, Fig. 3(B): Lorenz oscillator] having a larger control parameter is nearly close to zero, whereas that of the other unit remains significantly higher. When the values of the control parameters of both units have the same value, the values of the $\mathrm{CE}$ are the same and zero-complete synchronization state is obtained. Figures 3(C) and 3(D) plot the difference of the conditional entropies for the system of two Rössler oscillators and Lorenz oscillators, respectively. The figures underscore the rapid transition of the conditional entropies near the point when the control parameters of the two units are identical.

As it is expected in the case of phase synchronization, the fact that the phases of the two, coupled units are highly correlated does not correspond to synchronization of the coordinates of the two units. To illustrate this fact we define the synchronization error of the two units to be $E(t)$ $=\sqrt{\left(x_{1}(t)-x_{2}(t)\right)^{2}+\left(y_{1}(t)-y_{2}(t)\right)^{2}+\left(z_{1}(t)+z_{2}(t)\right)^{2}}$. Figures 4(A) and 4(B) depict synchronization error and its autocorrelation function of the two coupled, nonidentical Lo-

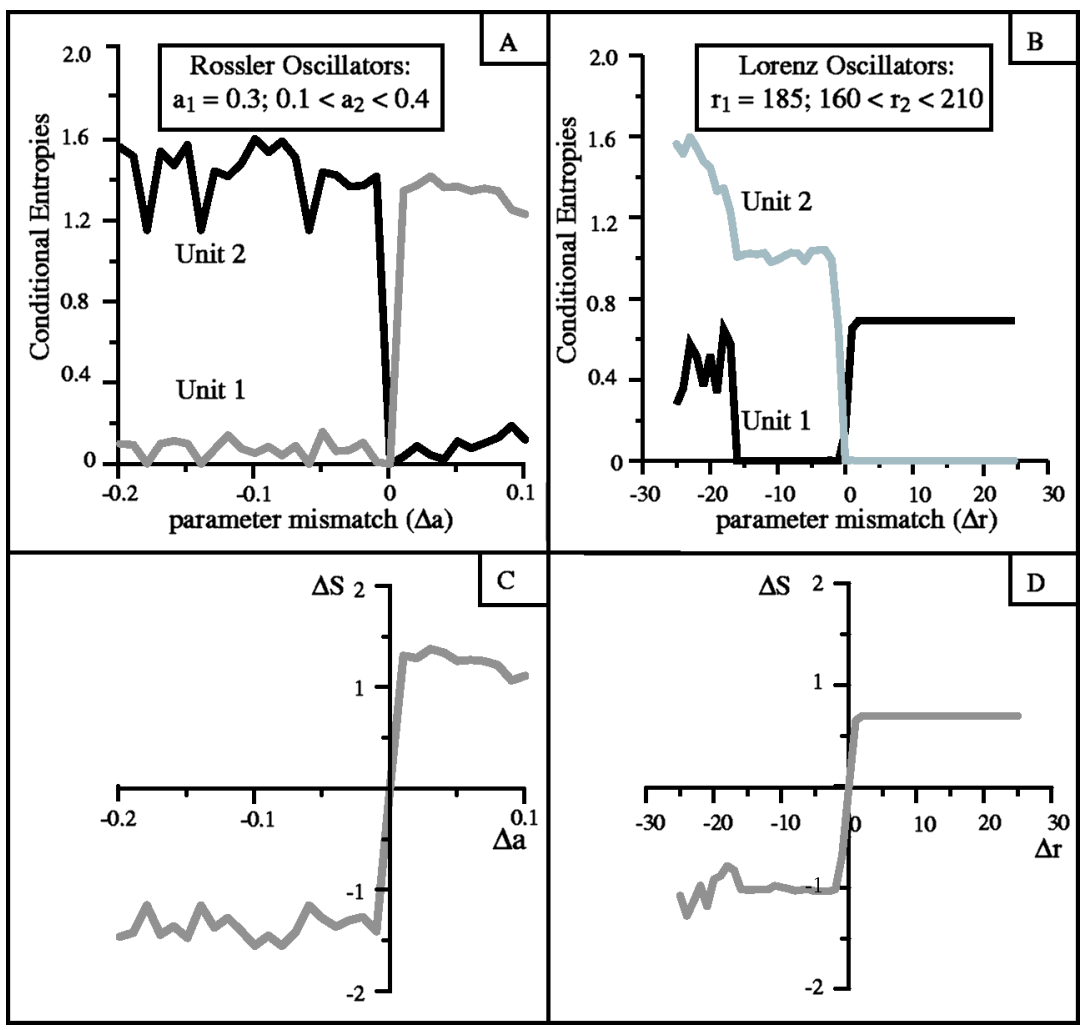

FIG. 3. Dependence of the conditional entropies on the parameter mismatch when no adaptation is present. The unit having larger value of the control parameter has much lower value of CE than that with lower parameter value: (A) two nonidentical Rössler oscillators, (B) Lorenz oscillators, (C) and (D) the difference of CEs is plotted as a function of the parameter mismatch to underscore abrupt transition around the point when the parameters are identical; (C) Rössler system and (D) Lorenz system. 


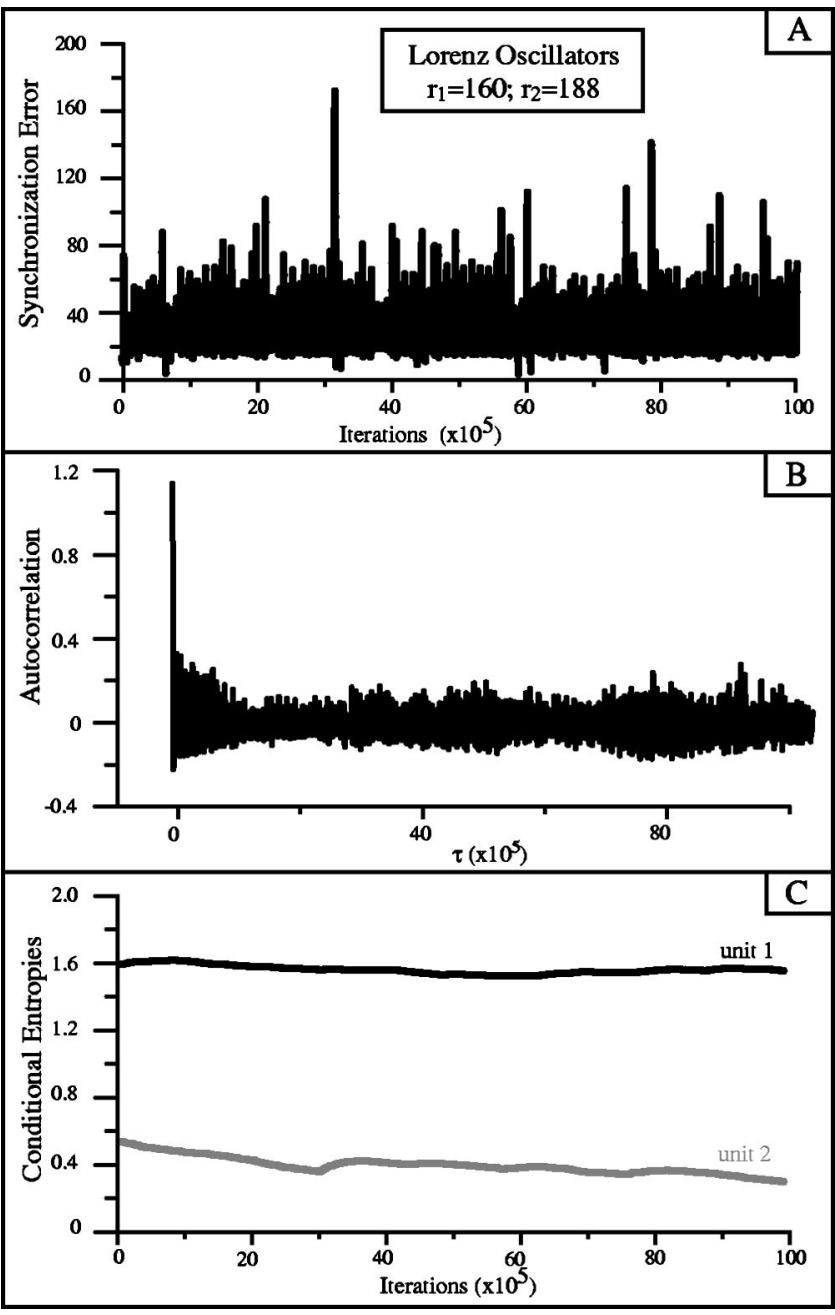

FIG. 4. Synchronization of two coupled nonidentical Lorenz oscillators: (A) synchronization error of the two units as a function of time; (B) autocorrelation function of the synchronization error; and (C) conditional entropies calculated during the same simulation. The autocorrelation function of the synchronization error rapidly converges but the significantly different CEs indicate phase synchronization of the two units.

renz oscillators. The autocorrelation function of the error rapidly converges to zero, indicating lack of synchronization between the amplitudes of their coordinates. The conditional entropies [Fig. 4(C)] remain significantly different, indicating a fixed phase dependence of the two coupled units.

As it will be shown below when the parameter adaptive control process is activated, the distributions of the interevent intervals change due the dynamic updating of the distributions in Eq. (1). This permits the use of the difference between the conditional entropies of the two units as a control mechanism of the adaptive process.

\section{ADAPTATION AND SYNCHRONIZATION USING PROPERTIES OF THE PHASE LAG DURING PHASE SYNCHRONIZATION}

To illustrate the feasibility of phase lag between the two units being the underlying mechanism during the adaptation and synchronization process, we studied the system of two coupled initially nonidentical Rössler and Lorenz oscillators. For both systems, one of the units had a constant value of the control parameter (we refer to it as the control unit), whereas the control parameter of the other unit, the target unit, was being modified during the adaptation process.

The equations of the system of two coupled, nonidentical Rössler oscillators are given by

$$
\begin{aligned}
& \dot{x}_{c, t}=-\left(z_{c, t}+y_{c, t}\right), \\
& \dot{y}_{c, t}=x_{c, t}+a_{c, t} y_{c, t}+\alpha\left(y_{t, c}-y_{c, t}\right), \\
& \dot{z}_{c, t}=b+\left(x_{c, t}-c\right) z_{c, t},
\end{aligned}
$$

where the subscript denotes the control and the target unit; $a_{c, t} \in[0.1,0.4], b=0.2$, and $c=10.0$ are the control parameters of the oscillators; and $\alpha=0.4$ is the coupling. The values of parameters $a_{c}$ and $a_{t}$ were different for both units. The events for the Rössler oscillator were defined as the times at which the oscillator's trajectory (unit 1 or 2) crossed a specified Poincaré section $(z=1)$, whereas the two coupled Lorenz oscillators are defined by

$$
\begin{aligned}
& \dot{x}_{c, t}=\sigma\left(y_{c, t}+x_{c, t}\right), \\
& \dot{y}_{c, t}=-x_{c, t} z_{c, t}+r_{c, t} x_{c, t}-y_{c, t}+\alpha\left(y_{c, t}-y_{t, c}\right), \\
& \dot{z}_{c, t}=x_{c, t} y_{c, t}-b z_{c, t},
\end{aligned}
$$

where $r_{c, t} \in[160,210]$ is the control parameter of the Lorenz oscillators and is initially different for both units. The values of other parameters were set to $\sigma=10$ and $b=\frac{8}{3}$. Each event was defined when the trajectory of a unit crossed the Poincaré section defined as $y=0$.

The adjustment of the control parameter of the target unit was linked to the difference of the CEs measured at both units $\left(S_{c}-S_{t}\right)$ [Figs. 3(C) and 3(D)]. Thus, the adaptation mechanism is based solely on the distributions of relative interevent intervals of the events appearing at both units and is not linked to the specific equations describing the dynamics of the system. For both systems the control parameter of the target was adjusted according to equations:

$$
\Delta \mathrm{CP}_{t}(t)=\beta\left(S_{c}-S_{t}\right),
$$

and

$$
\mathrm{CP}_{t}(t)=\mathrm{CP}_{t}(t-1)+\Delta \mathrm{CP}_{t}(t),
$$

where $\mathrm{CP}$ is the control parameter of the target $\left(a_{t}\right.$ or $r_{t}$ for Rössler and Lorenz oscillators respectively); and $\beta$ $=0.00001$ is a proportionality constant. The magnitude of $\beta$ determines the rate of adaptation (the convergence of the control parameter of the target) but is limited in its value by $\Delta P$. If the rate of change of $a_{t}$ or $r_{t}$ is too fast in comparison with the changes of the distributions of the timings [measured as $\left(S_{t}-S_{c}\right)$ ] the parameter adjustment will overshoot and oscillate around the value of the control parameter of the control unit. Note that Eq. (4) requires a monotonic relation between the differences of the conditional entropies and the parameter values. Thus the presented technique can be applied successfully only for the parameter regimes where the direction of the phase lag during phase synchronization of the two units remains constant. However, this constraint is significantly less restrictive than the one encountered in our earlier work. ${ }^{18}$ 


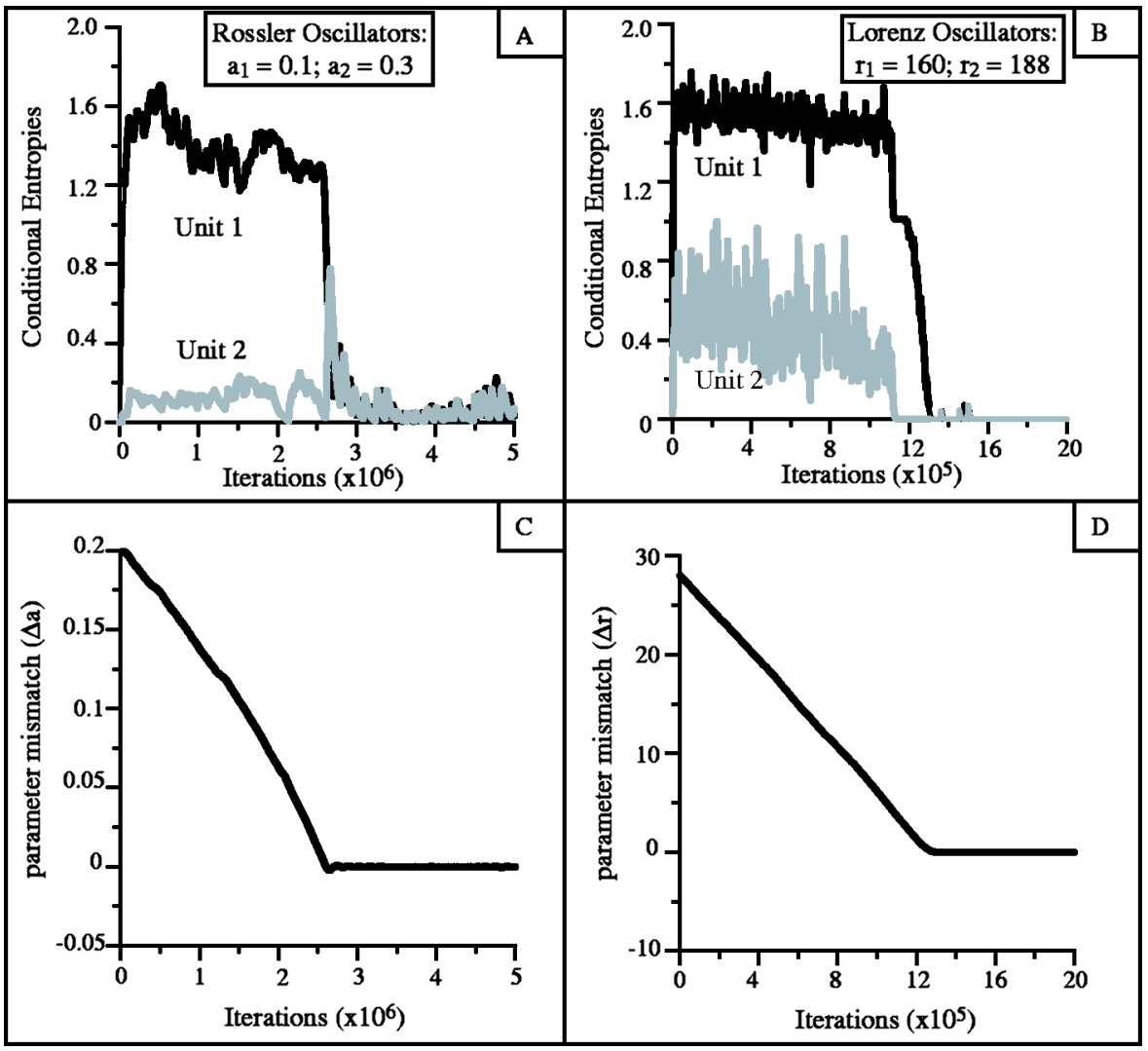

FIG. 5. Convergence of the target control parameter and changes of the $\mathrm{CE}$ difference during the adaptation process for Rössler and Lorenz systems. As the values of the target control parameter converges to that of the control the difference between $\mathrm{CE}$ rapidly decreases. (A) and (C) Difference of the entropies and the convergence of the control parameters for the Rössler system; (B) and (D) difference of the entropies and the convergence of the control parameters for Lorenz system.

The example of convergence of the control parameter of the target unit to the value of that of the control unit, together with the changes in the conditional entropies for both units during the adaptation process, is shown in Fig. 5. Figures 5(A) and 5(B) show the evolution of the CEs during the adaptation for the Rössler and Lorenz system, respectively, whereas Figs. 5(C) and 5(D) show the control parameter convergence for both systems during the same simulation. The control parameter of the target $\left(a_{t}, r_{t}\right.$ for Rössler and Lorenz oscillators, respectively) converges at a constant rate to that of the control unit ( $a_{c}, r_{c}$, respectively), as the values of the conditional entropies $S_{c}$ and $S_{t}$ remain relatively unchanged during the initial stages of the adaptation process, when the parameter mismatch is relatively high. This indicates the two units remain phase synchronized. However, when the control parameters of the control and target converge and their mismatch becomes small $\left(a_{t} \rightarrow a_{c}, r_{t} \rightarrow r_{c}\right)$, the difference in conditional entropies rapidly decreases $\left(\left|S_{c}-S_{t}\right| \rightarrow 0\right)$ and thus the adaptation process is halted. This is due to the fact that the two units became identical and achieved the state of complete synchronization in which the phase lag is consistently zero.

We tested the convergence of the control parameters during the adaptation process as a function of their initial values for both systems. We constructed a grayscale map spanning the ranges of the convergence values of the units' control parameters ( $a_{t, c}$ and $r_{t, c}$ for Rössler and Lorenz oscillators, respectively) in Fig. 6 . The values of the control parameters in both systems span chaotic as well as periodic regimes. The grayscale represents the time (in iteration steps) needed for the control parameter convergence of the two units. The simulation was halted when the synchronization error $E$ $\leqslant 0.001$ was achieved. Every pixel corresponds to six simulation runs that were initialized with different initial conditions $\left(x\left(t_{0}\right), y\left(t_{0}\right), z\left(t_{0}\right)\right)$ and averaged over symmetrical parameter pairs. In our simulations, the two units converged for any pair of initial values of the control parameters $\left[\left(a_{c}\left(t_{0}\right), a_{t}\left(t_{0}\right)\right)\right.$ and $\left(r_{c}\left(t_{0}\right), r_{t}\left(t_{0}\right)\right)$, respectively $]$. This indicates that the measurement of the properties of the phase lag provides a robust mechanism for driving the adaptation in the system of initially, nonidentical coupled units. The speed of convergence, as expected, depends linearly on the magnitude of the initial difference between the values of the control parameters of the control and target units, as the convergence rate does not depend on the magnitide of the phase lag and thus is approximately constant.

To further investigate the applicability of changing properties of the phase lag as a possible mechanism to control the parameter adaptation of the coupled, nonidentical units, we investigated the convergence of the mismatched control parameters as a function of noise amplitude. The term $A_{n} \xi(t)$ was added to the evolution of the $y$ coordinate of the control and target unit, where $\xi(t) \in[-1,1]$ was a random variable having uniform distribution. For both Lorenz and Rössler systems, the noise amplitude was varied so that the ratio between the noise amplitude and the maximal signal amplitude $\quad\left(A_{s}=\sqrt{\left(x_{\max }-x_{\min }\right)^{2}+\left(y_{\max }-y_{\min }\right)^{2}+\left(z_{\max }-z_{\min }\right)^{2}}\right)$, $A_{n} / A_{s} \in[0,0.25]$. The addition of noise widens the transition region of the conditional entropies around the point $\left(a_{c}=a_{t}\right)$ for both Rössler and Lorenz systems. This is illustrated in Fig. 7, where we calculated the conditional entro- 


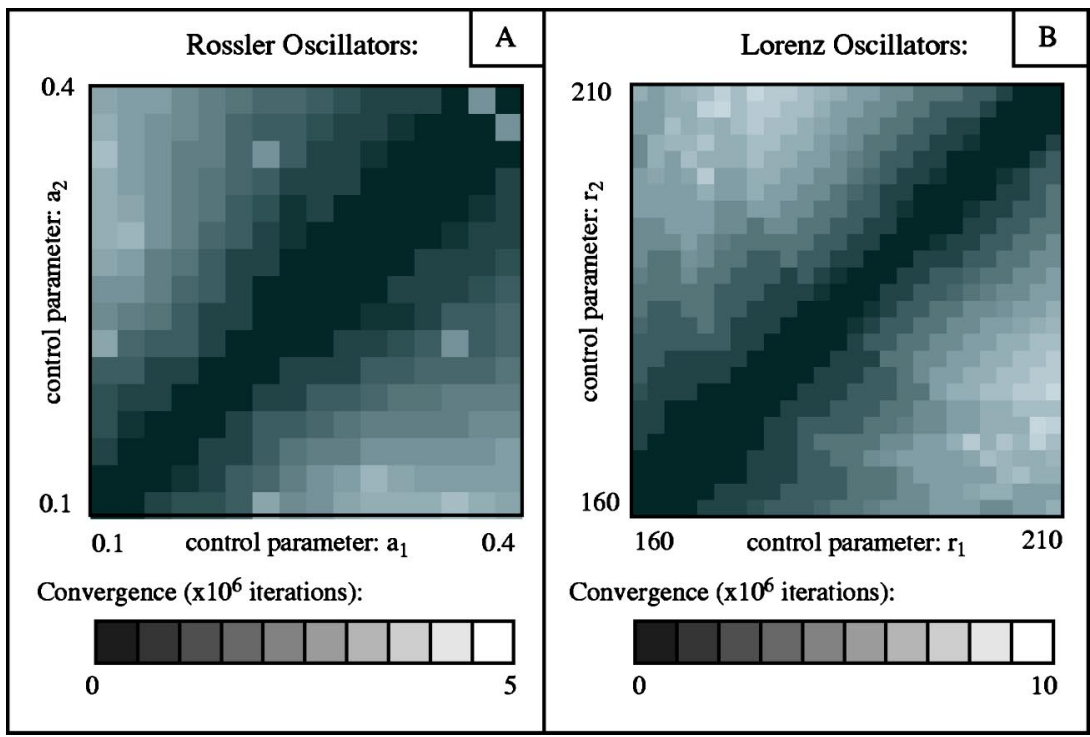

FIG. 6. Convergence of the control parameters for initially different parameter pairs $\left(a_{t, c} r_{t, c}\right)$. The grayscale denotes the time of convergence. Every point (pixel) is an average of six simulation runs taken over symmetrical parameter pairs. The time of convergence is dependent on the magnitude of the initial difference of the control parameter values for control and target units. The lowest and highest convergence time was 2000 and $10^{6}$ iterations, respectively, for Rössler system and 10000 and $6.5 \times 10^{6}$, respectively, for Lorenz system: (A) Rössler system and (B) Lorenz system.

pies for control and target for different magnitudes of parameter mismatch when adaptation is not present. The apparent widening [Figs. 7(A) and 7(C): Rössler system; Figs. 7(B) and 7(D): Lorenz system] is due to the fact that when the parameter mismatch is small the phase shift between the two synchronized units is relatively small and the noise significantly perturbs the relative order of the events of the control and target unit. However, the fact that the transition of the conditional entropies is widened does not significantly change the location of $S_{c}=S_{t}$ being at $a_{c}=a_{t}$ and therefore the adaptation based on the $S_{c}-S_{t}$ is still achieved. Additionally, we measured the speed of convergence as a function of $A_{n} / A_{s}$. Figure 8 plots the convergence rate of the control parameters as a function of the noise-to-signal ratio. Every point is an average of ten simulation runs. The calculation is initialized randomly, however the initial difference between control parameters between control and target units $\left[\left(a_{c}\right.\right.$ $\left.-a_{t}\right)$ and $\left(r_{c}-r_{t}\right)$, respectively] is kept constant for all simulation runs. It is considered that the convergence is achieved when the synchronization error is $E(t) \leqslant 0.01$. The convergence time increases significantly with the noise amplitude, however even for the values of $A_{n} / A_{s}=0.25$ convergence is still achieved.

Finally, we investigated the convergence speed of the

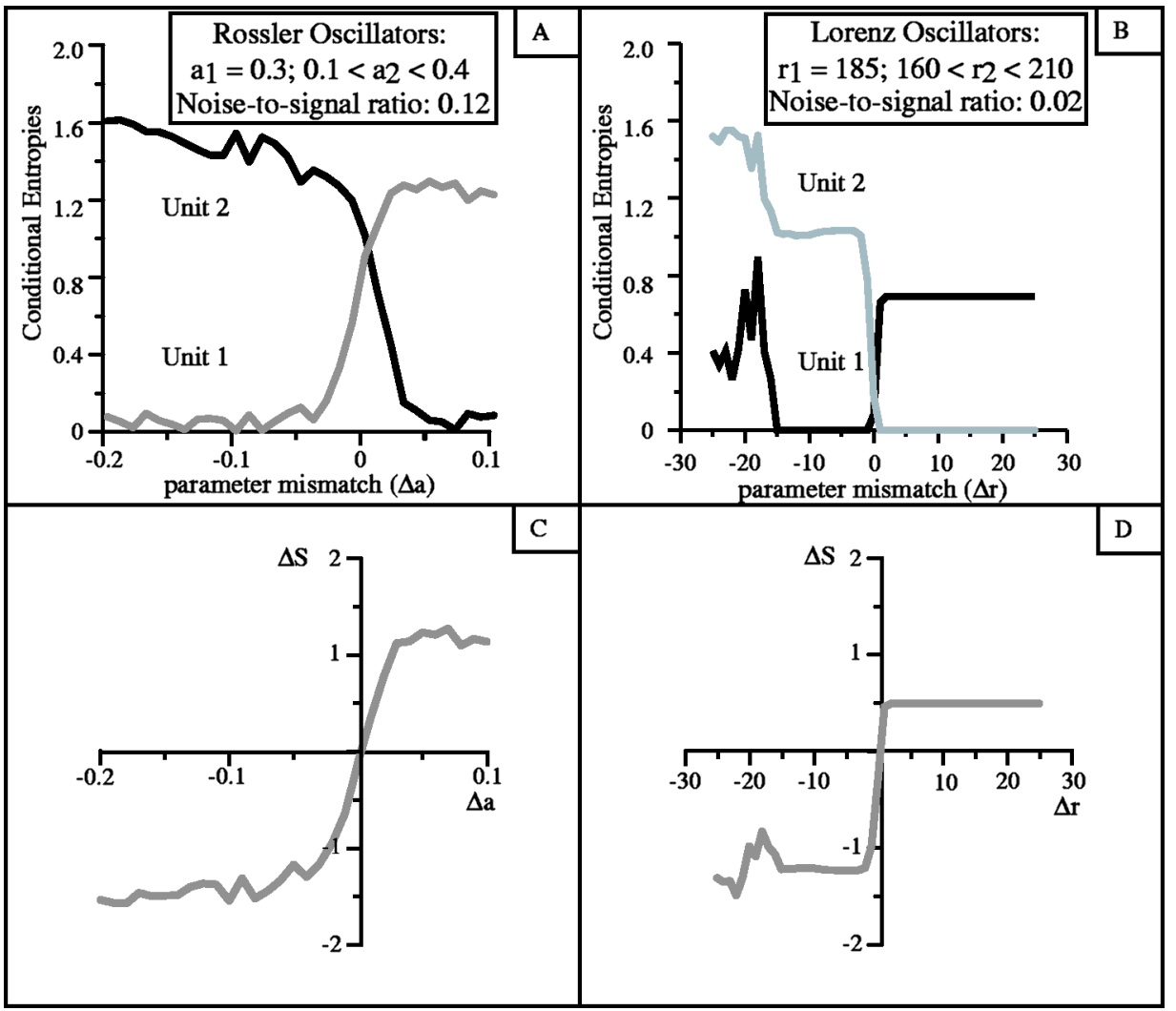

FIG. 7. Changes in CEs as a function of parameter mismatch in the presence of noise (no adaptation). The transition near the point $a_{c}=a_{t}, r_{c}=r_{t}$ becomes wider, however the values of CE remain the same at the point when the control parameters are identical: (A) Rössler system, (B) Lorenz system. (C) and (D) Difference of CEs shown on (A), (B), respectively. 


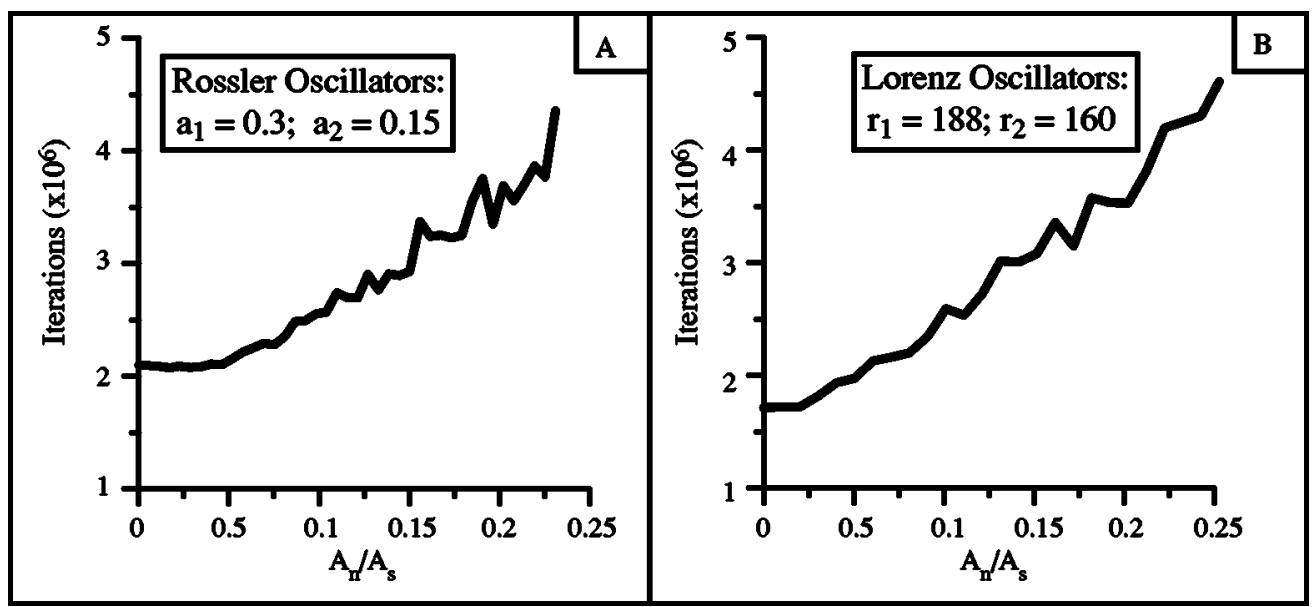

FIG. 8. The convergence time of the target control parameter as a function of the ratio $A_{n} / A_{s}$. The convergence time increases significantly for both systems: (A) Rössler system $(\beta=0.00004)$ and $(B)$ Lorenz system $(\beta=0.0075)$.

control parameters as a function of the coupling strength $\alpha$. As before, the convergence time is calculated from the start of the simulation until the synchronization error is $E(t)$ $\leqslant 0.001$. The parameter convergence is obtained even for relatively low coupling strengths (Fig. 9). The convergence time initially decreases rapidly with the coupling strength. It then stabilizes and remains constant for higher values of $\alpha$. The successful adaptation was obtained for $\alpha=0.15$ for the Rössler oscillators [Fig. 9(A)] and $\alpha=1.5$ for coupled Lorenz oscillators [Fig. 9(B)]. Every point on each graph is an average of four simulation runs.

\section{ADAPTATION OF NEURONAL ACTIVITY THROUGH MODIFICATION OF INTRINSIC CELL PROPERTIES}

We have shown above that the changing properties of the phase lag during phase synchronization of two nonidentical units can be used as a driving mechanism for parameter convergence in the coupled system. The parameter convergence causes the two units having initially different dynamical properties to become identical and achieve a complete synchronization state. Here, we show that such a mechanism of adaptation, based on temporal interdependencies of spike timings, can be easily extended to a system of coupled neurons and thus may lead to synchronization of neural activity in the brain. As an example, we use two coupled Hindmarsh-Rose models of thalamocortical neurons:

$$
\begin{aligned}
& \dot{x}_{c, t}=y_{c, t}-a x_{c, t}^{3}+b x_{c, t}^{2}-z_{c, t}+I_{0_{c, t}}+\alpha\left(x_{t, c}-x_{c, t}\right), \\
& \dot{y}_{c, t}=c-d x_{c, t}^{2}-y_{c, t}, \\
& \dot{z}_{c, t}=r\left[s\left(x_{c, t}-x_{0}\right)-z_{c, t}\right],
\end{aligned}
$$

where $a=1.0, b=3.0, c=1.0, d=5.0, r=0.006, s=4.0$, and $x_{0}=-1.6$ are the parameters of the model and $\alpha=1.1$ is the coupling strength. As before, the subscripts $c, t$ correspond to the control and target neurons, respectively. The parameter $I_{0}$ represents the amplitude of external current applied to the neuron and is the control parameter of the units.

As before, the relative timings of the action potentials of the two neurons are used to construct the appropriate distributions and to calculate the CEs for both neurons and the difference in those entropies, $\left(S_{c}-S_{t}\right)$ is used to adjust the control parameter of the target neuron. The adaptation and complete synchronization is achieved relatively quickly (Fig. 10), indicating that the relative timings of the action potentials can be used to determine the internal changes required to adjust the firing patterns of the neurons to achieve the synchronized state. Figure 10(A) plots the magnitude of the

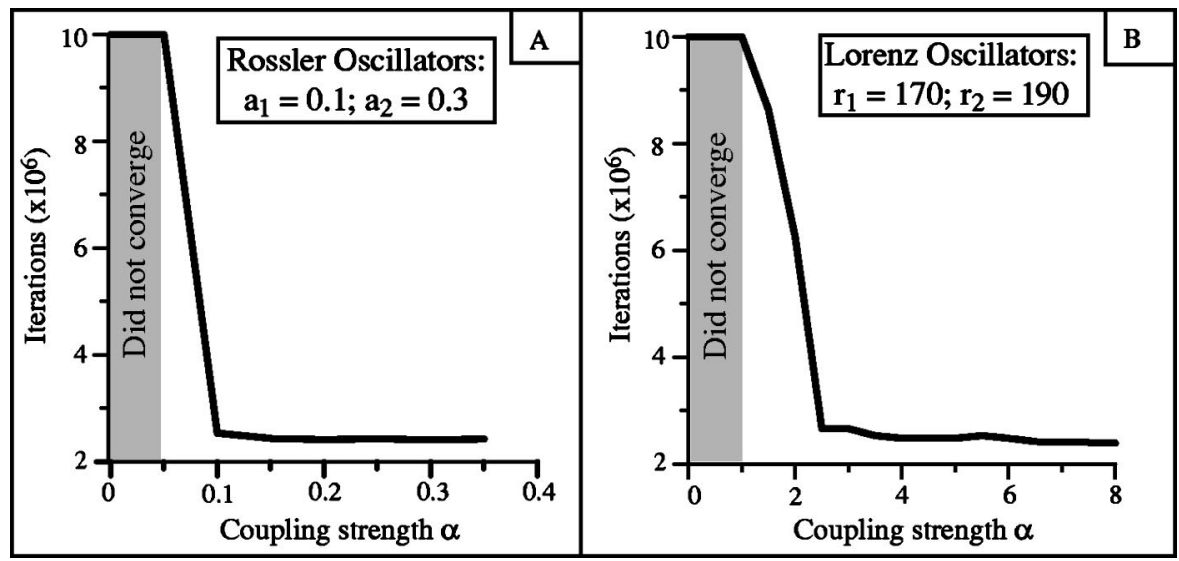

FIG. 9. The convergence time of the target control parameter as a function of the coupling strength. The successful adaptation was obtained for the value of the coupling as low as $\alpha$ $=0.15$ for the Rössler system (A) and $\alpha=1.5$ for coupled Lorenz system (B). 


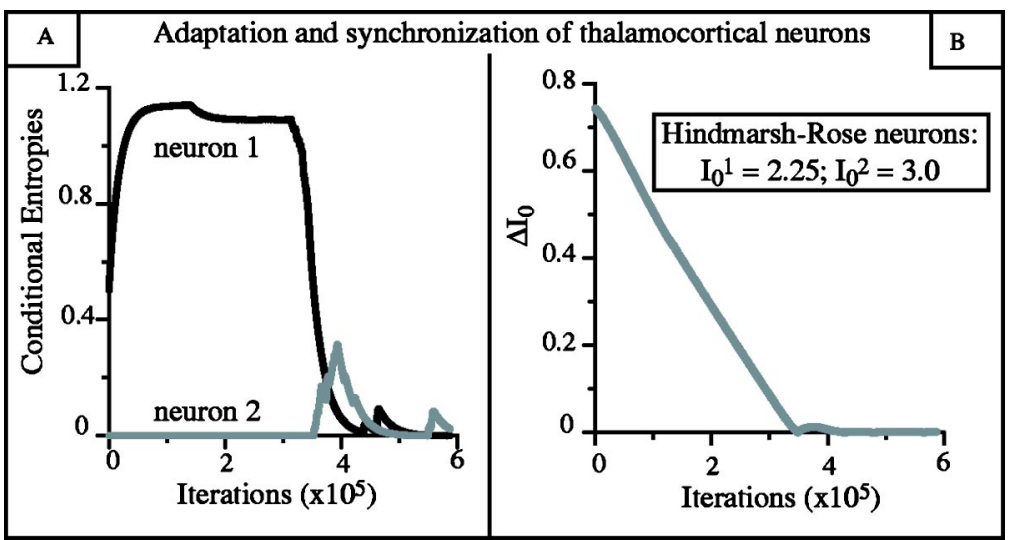

FIG. 10. Adaptation of two thalamocortical neurons based on the properties of the distributions of relative spike timings obtained via $\mathrm{CE}$ estimation: (A) changes of CEs of both neurons during the adaptation process and (B) convergence of the control parameters. entropy difference of the two neurons, whereas Fig. 10(B) plots the magnitude of the control parameter mismatch. The target control parameter converges quickly to that of the control neuron-the two neurons synchronize after a brief adaptation of the target neuron. Thus the information about the relative timing of the spike firing of the two cells is (at least theoretically) enough to control the adaptation of their properties to obtain synchronization.

\section{CONCLUSIONS}

We have shown that the properties of the distributions of the relative event timings can be used to control the parameter adaptation process in a system of two coupled, nonidentical units. This is due to the fact that the mismatch of the control parameters causes the nonidentical coupled units to achieve phase synchronization with a phase lag depending on their relative frequencies. Based on the latter finding we have defined a measure that monitors the changes of the relative distributions of the interevent lengths by calculating the difference of entropies of both distributions.

The crucial advantage of this adaptation scheme is that the information used to control the convergence of initially different units is based on the properties of the time series of their relative event timings that can be observed experimentally and does not use any information about the mathematical formulation of the units' dynamics.

We applied the proposed adaptation scheme to systems of two coupled, nonidentical Rössler oscillators as well as to two nonidentical Lorenz oscillators and have shown that for both systems the proposed adaptation scheme is robust as the two initially nonidentical units converge over a wide range of parameter spanning periodic as well as chaotic behavior of the control and target. Moreover, the proposed mechanism of adaptation is relatively robust in the presence of noise.

The proposed parameter adaptive control may possibly be applied to systems of multiple units. The parameter changes in such systems can be driven by the pairwise assessment of the mutual phase interdependency between the individual units, or by assessment of the phase relation between the individual unit and its mean coupling signal.

Finally, we show that the same adaptation mechanism can be used to control the adaptation of a system of the thalamocortical neurons. The target neuron modifies the magnitude of external input current to achieve complete syn- chronization with the control neuron. One can also foresee application of other control variables as it is established that the neural excitation could be controlled intrinsically by neurons through the expression of appropriate receptors. ${ }^{30,31}$

${ }^{1}$ L. M. Pecora and T. L. Carroll, "Synchronization in chaotic systems," Phys. Rev. Lett. 64, 821 (1990).

${ }^{2}$ L. Kocarev and U. Parlitz, "Synchronizing spatiotemporal chaos in coupled nonlinear oscillators," Phys. Rev. Lett. 77, 2206 (1996).

${ }^{3}$ L. Kocarev and U. Parlitz, "Generalized synchronization, predictability, and equivalence of unidirectionally coupled dynamical systems," Phys. Rev. Lett. 76, 1816 (1996).

${ }^{4}$ N. F. Rulkov, M. M. Suschik, L. S. Tsimring, and H. D. I. Abarbanel, "Generalized synchronization of chaos in directionally coupled chaotic systems," Phys. Rev. E 51, 980 (1995).

${ }^{5}$ U. Ernst, K. Pawelzik, and T. Geisel, "Synchronization induced by temporal delays in pulse-coupled oscillators," Phys. Rev. Lett. 74, 1570 (1995).

${ }^{6} \mathrm{~W}$. Gerstner, "Rapid phase locking in systems of pulse-coupled oscillators with delays," Phys. Rev. Lett. 76, 1755 (1996).

${ }^{7}$ U. Parlitz, L. Junge, W. Lauterborn, and L. Kocarev, "Experimental observation of phase synchronization," Phys. Rev. E 54, 2115 (1996).

${ }^{8}$ K. Sauvé, "Gamma-band synchronous oscillations: Recent evidence regarding their functional significance," Conscious Cogn 8, 213 (1999).

${ }^{9}$ W. Singer, "Neuronal synchrony: a versatile code for the definition of relations?" Neuron 24, 49 (1999).

${ }^{10}$ E. Başar, Brain Function and Oscillations. I. Brain OscillationsPrinciples and Approaches (Springer, Berlin, 1998).

${ }^{11} \mathrm{C}$. M. Gray, "The temporal correlation hypothesis of visual feature integration: still alive and well," Neuron 24, 31 (1999).

${ }^{12}$ R. Eckhorn, R. Bauer, W. Jordan, M. Brosh, W. Kruse, M. Munk, and H. J. Reitboeck, "Coherent oscillations: a mechanism of feature linking in the visual cortex?" Biol. Cybern. 60, 121 (1988).

${ }^{13}$ Y. Lam, L. B. Cohen, M. Wachowiak, and M. Zochowski, "Odors elicit three different oscillations in the turtle olfactory bulb," J. Neurosci. 20, 749 (2000).

${ }^{14}$ A. Luthi and D. A. McCormick, "H-current: properties of a neuronal and network peacemaker," Neuron 21, 9 (1998).

${ }^{15}$ C. Zhou and C.-H. Lai, "Decoding information by following parameter modulation with parameter adaptive control," Phys. Rev. E 59, 6629 (1999).

${ }^{16}$ T.-L. Liao and S.-H. Tsai, "Adaptive synchronization of chaotic systems and its application to secure communications," Chaos, Solitons Fractals 11, 1387 (2000).

${ }^{17}$ A. d'Anjou, C. Sarasola, F. J. Torrealdea, R. Orduna, and M. Grana, "Parameter-adaptive identical synchronization disclosing Lorenz chaotic masking," Phys. Rev. E 63, 046213 (2001).

${ }^{18}$ M. Żochowski and R. Dzakpasu, "Adaptation of nonlinear systems through dynamic entropy estimation," J. Phys. A 37, 2223 (2003).

${ }^{19}$ M. Żochowski and R. Dzakpasu, "Condtional entropies; phase synchronization and changes in the directionality of information flow in neural systems," J. Phys. A 37, 3823 (2004). 
${ }^{20}$ O. E. Rössler, “An equation of continuous chaos,” Phys. Lett. A 57, 397 (1976).

${ }^{21}$ E. N. Lorenz, "Deterministic nonperiodic flow," J. Atmos. Sci. 20, 130 (1963).

${ }^{22}$ J. L. Hindmarsh and R. M. Rose, "A model for rebound bursting in mammalian neurons," Philos. Trans. R. Soc. London, Ser. B 346, 129 (1994).

${ }^{23}$ J. L. Hindmarsh and R. M. Rose, "A model of intrinsic and driven spindling in thalamocortical neurons," Philos. Trans. R. Soc. London, Ser. B 346, 165 (1994).

${ }^{24}$ J. L. Hindmarsh and R. M. Rose, "Resonance in a model of a mammalian neuron," Philos. Trans. R. Soc. London, Ser. B 346, 151 (1994).

${ }^{25}$ M. G. Rosenblum, A. S. Pikovsky, and J. Kurths, "From phase to lag synchronization in coupled chaotic oscillators," Phys. Rev. Lett. 78, 4193 (1997).

${ }^{26}$ M. G. Rosenblum, A. S. Pikovsky, and J. Kurths, "Phase synchronization in chaotic oscillators," Phys. Rev. Lett. 76, 1804 (1996).

${ }^{27}$ M. G. Rosenblum, A. S. Pikovsky, G. Osipov, and J. Kurths, "Phase synchronization of chaotic oscillators by external driving," Physica D 104, 219 (1997).

${ }^{28}$ C. Zhou, J. Kurths, I. Z. Kiss, and J. L. Hudson, "Noise-enhanced phase synchronization of chaotic oscillators," Phys. Rev. Lett. 89, 014101 (2002).

${ }^{29}$ D. Pazo, A. M. Zaks, and J. Kurths, "Role of unstable periodic orbits in phase and lag synchronization between coupled chaotic oscillators," Chaos 13, 309 (2003).

${ }^{30}$ N. S. Desai, G. G. Turrigiano, and L. C. Rutherford, "Plasticity in the intrinsic excitability of cortical pyramidal neurons," Nat. Neurosci. 2, 515 (2002).

${ }^{31}$ M. N. Shadlen and J. A. Movshon, "Synchrony unbound: A critical evaluation of the temporal binding hypothesis," Neuron 24, 67 (1999). 